\title{
Soil moisture retrieval under wheat crop using RISAT-1 hybrid polarimetric SAR data
}

\author{
P.K. SHARMA ${ }^{1,2 *}$, D. KUMAR ${ }^{2}$, H. S. SRIVASTAVA ${ }^{1}$, P. PATEL $^{3}$ and T. SIVASANKAR ${ }^{1}$ \\ ${ }^{1}$ Indian Institute of Remote Sensing, ISRO, Dehradun (Uttarakhand) - 248001 \\ ${ }^{2}$ Indian Institute of Technology (ISM), Dhanbad (Jharkhand) - 826004 \\ ${ }^{3}$ Space Applications Centre, Ahmedabad (Gujarat) - 380015 \\ *Corresponding author: pavanks.space@gmail.com
}

\begin{abstract}
The study aims to retrieve soil moisture from RISAT-1 hybrid polarimetric SAR data. Although the use of linear polarimetric SAR data has been well understood and documented, but hybrid polarimetric SAR data is grossly under explored and under reported for this purpose. Regression analysis has been carried to develop soil moisture retrieval models and validated the same. The retrieval models have been developed from back scattering coefficients $\left(\sigma_{\mathrm{RH}} \& \sigma_{\mathrm{RV}}\right)$ and $\mathrm{m}-\delta$ space decomposition parameters (even bounce, odd bounce, and volume component) generated from RISAT-1 hybrid polarimetric SAR data. A total of three models are analyzed in this work, (i) using both $\sigma_{R H} \& \sigma_{R V}$, (ii) volume component, and (iii) using even bounce, odd bounce and volume component. The study results showed that the model using $\mathrm{m}-\delta$ decomposition derived parameters can provide better accuracy with $\mathrm{R}^{2}$ and RMSE of 0.92 and 2.45 per cent respectively in comparison to other two models.
\end{abstract}

Keywords : Hybrid polarimetry,m-delta decomposition,RISAT-1 SAR, soil moisture, wheat crop

The knowledge of spatial and temporal distribution of soil moisture is highly desirable for agricultural applications like precision farming, irrigation scheduling and yield estimation in combination with hydrological, meteorological, and ecological applications. Although several advanced ground-based instruments are available to estimate actual soil moisture, but it is laborious and expensive for measurement of large agricultural area and it provides point measurements of soil moisture which are too sparse to represent the soil moisture of surrounding area. Remote sensing becomes as better alternative for soil moisture monitoring by providing high spatial and temporal resolution data from local to global scales. Out of the total range of electromagnetic spectrum, microwave region is more suitable for soil moisture studies due to the large difference between the dielectric constant of water $(\approx 80)$ and that of dry soil $(\approx 3)$ at microwave frequencies (Srivastava et al., 2006). Several operational sensors are available in both active and passive modes operating in microwave region of electromagnetic spectrum for soil moisture studies. The data from passive microwave sensors is highly influenced by the vegetation than the soil moisture in vegetation covered areas (Patel and Srivastava, 2015). Coming to the active microwave remote sensing, the return signal is also sensitive to surface roughness and texture over bare agriculture fields and to crop cover over crop covered agriculture fields along with soil moisture (Sivasankar et al., 2018). Due to its penetration capability and high spatial resolution, SAR has become an important tool for soil moisture studies. But it needs to minimize the crop cover effect within the resolution cell to retrieve underneath soil moisture from crop covered fields (Gherboudj et al., 2011; Srivastava et al., 2015).

The SAR sensor parameters like frequency, incidence angle and polarization plays an important role to enhance the sensitivity of SAR data for a specific application. The penetration depth varies with the frequency of operation, higher the penetration depth for high frequency signals. Srivastava (2007) suggested the high frequency SAR data for deeper layer soil moisture studies and low frequency data for surface soil moisture studies. The increase in incidence angle makes the signal to propagate longer distance through the crop cover due to increase in slant range over crop covered agriculture fields. Whereas, low incidence angle SAR data is more sensitive towards underneath soil moisture over crop covered fields. The illuminated and return signals polarization is also plays an important role in soil moisture retrieval. So far linear polarized signal illuminated data have been used for soil moisture studies by several researchers (Dong et al., 2013; Srivastava 
et al., 2002). Srivastava et al., (2009) described the methodology to retrieve soil moisture by incorporating the effect of surface roughness and texture over bare agriculture fields and crop covered agriculture fields using linear polarized data. However, researchers found several technical and maintenance limitations in linear polarimetry SAR onboard space-borne platform (Patel et al., 2012). Advancement of hybrid polarimetry, which involves circular polarization transmission and receives coherent orthogonal linear polarizations, designed in such a way to overcome the limitations of linear polarimetry like Faraday rotation effect, available over only restricted swaths, more power requirement etc. Refer Raney (2007), for detailed description of hybrid polarimetry SAR architecture and its importance in earth observation missions. Indian Space Research Organization (ISRO) was launched an operational hybrid polarimetry SAR onboard Radar Imaging Satellite-1 (RISAT1), the first earth observing space-borne platform acquiring hybrid polarimetric SAR images, in 2012 to make use of hybrid polarimetric SAR data. Later, ISRO team has carried out an extensive research work to calibrate and validate the RISAT-1 hybrid polarimetric SAR data (Mishra et al., 2015). On the other hand, numerous researchers have been intensively using the RISAT- 1 hybrid polarimetric SAR data for various remote sensing applications including crop and soil moisture studies (Uppala et al., 2015;Srivastava et al., 2018a).

The capabilities of polarimetric parameters have proven better than backscattering coefficients for various applications. It has been reported by Sivasankar et al., (2015) that the hybrid polarimetric parameters (even bounce, odd bounce and volume component) are having more information about land cover targets than the backscattering coefficients $\left(\sigma_{\mathrm{RH}}^{\circ}, \sigma_{\mathrm{RV}}^{\circ}\right)$ generated from hybrid polarimetric SAR data. On the basis of these studies, here it is investigated the sensitivity of hybrid polarimetric parameters generated from RISAT-1 hybrid polarimetric SAR data (RH, RV) towards soil moisture over wheat crop covered agricultural fields. The detailed study methodology and results are discussed in the following sections.

\section{MATERIALS AND METHODS}

\section{Dataset and study area}

Current study carried out using RISAT-1 hybrid polarimetric SAR data acquired on March $2^{\text {nd }}, 2013$ over parts of Haridwar district, India. The study area is a relatively flat agricultural region located at $29^{\circ} 58^{\prime} \mathrm{N}$, latitude, $78^{\circ} 13^{\prime}$ $\mathrm{E}$, longitude. The detailed SAR sensor and data specifications are given in Table 1. In addition to the SAR data, Landsat data acquired on March $2^{\text {nd }}, 2013$ has been used for georeferencing and to cross check the fields' boundaries on SAR image. One major river, Ganga is flowing from northeast direction to southeast direction in the study area. Due to this, a large amount of study area is covered with irrigation. The study area is dominated with wheat and sugarcane agricultural crops during the image acquisition.

\section{Ground truth data collection}

A synchronous detailed ground truth data collection has been carried over study area for actual soil moisture readings. The quantitative number of fields and field specifications (should be $22 \times 22 \mathrm{~m}^{2}$ for FRS- 1 mode of RISAT-1) were considered as suggested in Patel and Srivastava (2013), to account the characteristic-fading phenomenon of SAR signal. The complete route plan and field boundaries were recorded using Garmin GPS. Frequency domain radiometry (FDR) probe method has been used to estimate surface soil moisture (volumetric, \%) in the 40 wheat crop agricultural fields with 5 samples from each field. Out of 40 samples, 10 randomly selected samples were chosen for model validation and the remaining 30 samples were used for model development.

\section{SAR data processing}

The backscattering coefficients $\left(\sigma_{\mathrm{RH}}\right.$ and $\left.\sigma_{\mathrm{RV}}\right)$ were generated using PolSDP software from RISAT-1 hybrid polarimetric SAR data, which was supplied by National Remote Sensing Centre. After conversion of DN to backscattering coefficients, enhanced Lee-filtering algorithm was used to suppress the speckle noise. And then, polarimetric parameters viz. even bounce, odd bounce and volume component were generated based on $m-\delta$ space decomposition. Further details about backscattering coefficients and $m-\delta$ space decomposition technique are given in Srivastava et al., (2018b). The hybrid polarization backscatter can be related with backscattering coefficients generated from linear polarimetric data as given in equation (1).

$\left[\begin{array}{l}S_{R H} \\ S_{R V}\end{array}\right]=\frac{1}{\sqrt{2}}\left[\begin{array}{c}S_{H H}-i S_{H V} \\ -i S_{V V}+S_{H V}\end{array}\right]$

Where, $\mathrm{S}_{\mathrm{xy}}$ indicates the complex backscatter observed when signal transmitted in ' $x$ ' polarization and received in ' $y$ ' polarization, ' $H$ ' represents the horizontal polarization, ' $\mathrm{V}$ ' indicates vertical polarization.

The hybrid polarimetric parameters and backscattering coefficients were stacked into a single file 
Table 1: SAR sensor and data specifications used in this

\begin{tabular}{ll}
\multicolumn{1}{c}{ study } & Details \\
\hline Parameter & C-band $(5.35 \mathrm{GHz})$ \\
\hline Frequency & $25.31 \mathrm{U}$ \\
Incidence angle & FRS-1 \\
Mode & Right Circular \\
Tx. polarization & Vertical \& Horizontal \\
Rx. polarization & $3 \mathrm{~m}$ \\
Azimuth resolution & $2 \mathrm{~m}$ \\
Range resolution & $30 \mathrm{~km}$ \\
Swath width &
\end{tabular}

and geo-referenced using Landsat image. The SAR signatures from appropriate fields were extracted with the help of the vector layer of route plan and sampled field boundaries. The generated backscattering coefficients $\left(\sigma_{\mathrm{RH}}^{\circ}\right.$ and $\left.\sigma_{\mathrm{RV}}^{\circ}\right)$ and polarimetric parameters (even bounce, odd bounce and volume component) are related with in-situ volumetric soil moisture as given in equations (2), (3), and (4).

$$
\begin{aligned}
& \text { SoM }=A+B * \sigma_{R H}^{\circ}+C * \sigma_{R V}^{\circ} \\
& \text { SoM }=A+B *(\text { Volumecomponent }) \\
& \text { SoM }=A+B *(\text { Evenbounce })+C *(\text { Oddbounce })+D * \text { (Volumecomponent })
\end{aligned}
$$

\section{RESULTS AND DISCUSSION}

It is known fact that the depolarization results due to multiple reflections within the vegetation volume from crop covered fields. So, Srivastava et al., (2006) has developed an empirical model using cross-polarized (HV) backscatter to incorporate the effect of crop cover along with like polarization (VV) for soil moisture retrieval. The study results observed the $\mathrm{R}^{2}$ of 0.92 and RMSE of 1.49 for developed model to estimate soil moisture from Bajra/Jowar crop covered fields. Whereas the hybrid polarization backscatter is a combination of like and cross polarized SAR backscatter as given in equ. (1). This indicates that the hybrid polarized backscatter is combined effect of crop cover as well as underneath soil moisture. However, an attempt has been made to retrieve soil moisture from RH and $\mathrm{RV}$ backscatter using empirical regression analysis like as mentioned in equ. (2).

The model coefficients ' $\mathrm{A}$ ', ' $\mathrm{B}$ ' and ' $\mathrm{C}$ ' are optimized based on least square method using in-situ surface soil moisture and RH, RV backscatter coefficients signatures from 30 farmers' fields. The coefficient of determination $\left(\mathrm{R}^{2}\right)$ of 0.82 observed for this model. This has been validated with sample size of 10 fields independent to the development set of samples and observed the RMSE of 4.09 per cent. As anticipated from the literature, the hybrid polarized backscatter coefficients have shown poor performance to retrieve soil moisture under wheat crop due to its inability to incorporate the crop cover effect.

It is well documented that the target decomposition can better represent the target properties than the backscattering coefficients of hybrid polairmetric SAR data (Sivasankar et al., 2018).Hence, $\mathrm{m}-\delta$ decomposition developed by Raney, (2007) has been used to estimate the even bounce, odd bounce and volume component from hybrid polarimetric SAR data. The volume component is proportional to un-polarized component of return signal, which results when the incident microwave signal interacts with a dielectric discontinue medium. Since, the significant amount of radiation at low incidence angles interacts with underneath soil by penetrating through the crop cover. Volume component has been assumed as sensitive variable to represent the underneath soil moisture, as water content in the soil acts as dielectric discontinue medium. Because of this, present study investigated the sensitivity of volume component towards soil moisture under wheat crop using empirical regression analysis, as given in equation (3).This model observed $\mathrm{R}^{2}$ of 0.87 with surface soil moisture and volume component signatures extracted from 30 farmers' fields and RMSE of 3.57 per cent observed with the sample size of 10 fields. This indicates that the volume component at low incidence angles can better represent soil moisture under wheat crop than the backscattering coefficients generated from hybrid polaimetric SAR data.

The un-polarized component of the return signal observed at an incidence angle has combined effect of both crop cover and underneath soil moisture (Srivastava et al., 2018 a \& b). However it is more sensitive towards underneath soil moisture at low incidence angles and crop cover at high incidence angles. Therefore, an attempt has been made by considering even bounce and odd bounce to incorporate the crop cover effect in the soil moisture model using volume component, as given in equation (4). This model observed the $\mathrm{R}^{2}$ of 0.92 from the development set of samples and RMSE of 2.45 per cent from the validation set of samples. The improvement of $R^{2}$ to 0.92 for (Eq. -4 ) to $R^{2}$ of 0.82 for (Eq.2) and decrease in RMSE to 2.45 per cent for (Eq.4) from 4.09 per cent for (Eq.2), represents that the target decomposition can better incorporate the effect of crop 
Table 2: Results of model analysis and validation for wheat covered fields

\begin{tabular}{|c|c|c|c|c|}
\hline \multirow[b]{2}{*}{ Model } & \multicolumn{2}{|c|}{ Model development } & \multicolumn{2}{|c|}{ Model validation } \\
\hline & $\mathrm{N}$ & $\mathrm{R}^{2}$ & $\mathrm{~N}$ & RMSE \\
\hline $\mathrm{SM}=\mathrm{A}+\mathrm{B} * \sigma_{\mathrm{RH}}+\mathrm{C} * \sigma_{\mathrm{RV}}$ & 30 & 0.82 & 10 & 4.09 \\
\hline $\mathrm{SM}=\mathrm{A}+\mathrm{B} *$ Volume Component & 30 & 0.87 & 10 & 3.57 \\
\hline $\begin{aligned} \mathrm{SM}=\mathrm{A} & +\mathrm{B} *(\text { Odd Bounce })+\mathrm{C} *(\text { Even Bounce }) \\
& +\mathrm{D} *(\text { Volume Component })\end{aligned}$ & 30 & 0.92 & 10 & 2.45 \\
\hline
\end{tabular}

Where A, B, C and D represents coefficients; $\mathrm{N}$ indicates the number of samples

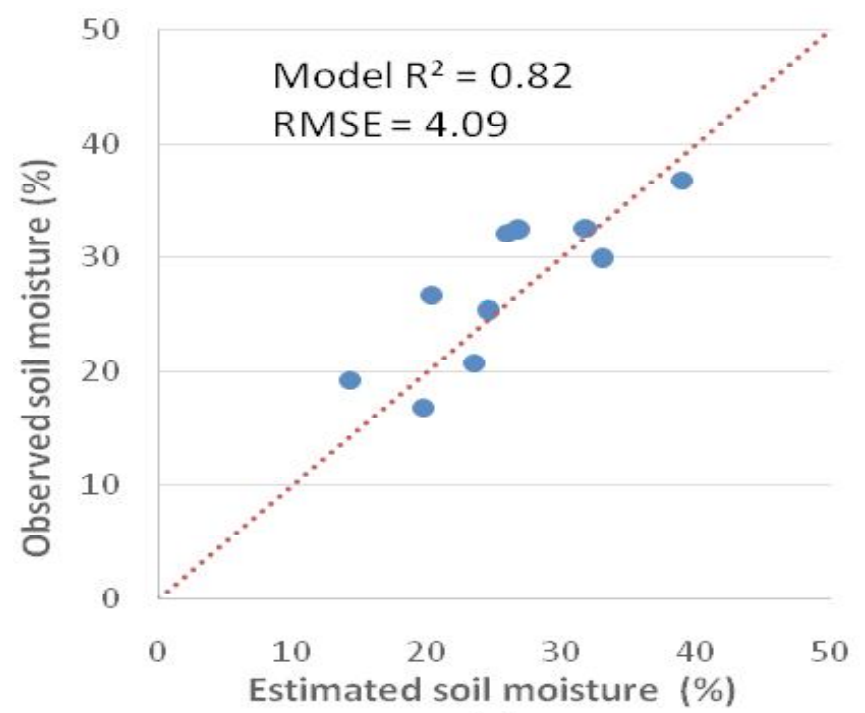

(a)

Fig.1: Scatter plot of the observed soil moisture vs the estimated soil moisture from backscattering coefficients.

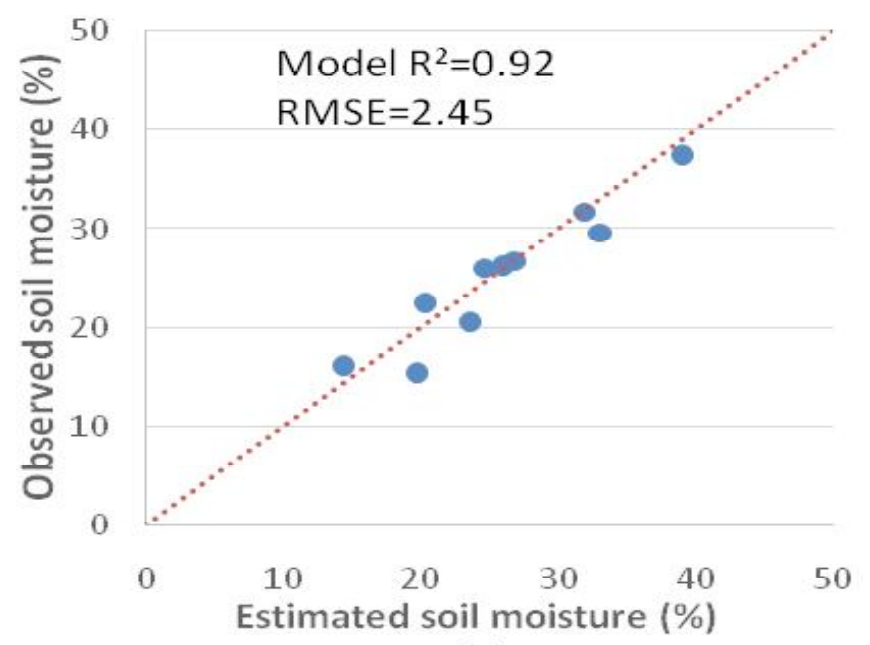

(c)

Fig. 3: Scatter plot of the observed soil moisture vs the estimated soil moisture from $\mathrm{m}-\delta$ space decomposition derived parameters (even bounce, odd bounce and volume component)

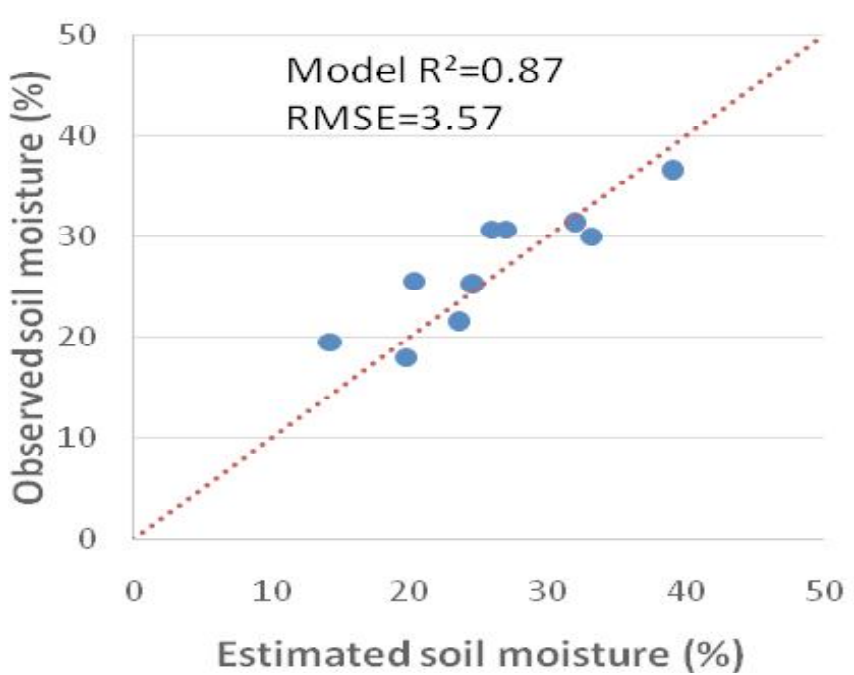

(b)

Fig. 2: Scatter plot of the observed soil moisture vs the estimated soil moisture from volume component

cover than the backscattering coefficients generated from hybrid polarimetric SAR data. The scatter plot of observed vs estimated soil moisture from equation (2), (3) and (4) are given in Fig. 1, 2 and 3 respectively.

\section{CONCLUSIONS}

This study has been carried out to evaluate the capability of hybrid polarimetric SAR data for soil moisture retrieval over wheat crop covered agricultural fields. In this context, the sensitivity of backscattering coefficients $\left(\sigma_{\mathrm{RH}}^{0}\right.$, $\sigma_{\mathrm{RV}}^{0}$ ) and target decomposition generated from hybrid polarimetric SAR data were analysed towards the water content in the Earth's material. The study results successfully demonstrated that the proper use of polarimetric parameters (even bounce, odd bounce and volume component) can significantly enhance the accuracy of soil moisture retrieval than the backscattering coefficients generated from RISAT- 1 hybrid polarimetric SAR data. It is also observed that, the volume component at low incidence 
angle SAR data is highly sensitive to the water content present in the Earth's material under crop covered agriculture fields due to the increase in dielectric discontinuity with soil moisture. The present study has considered the empirical regression approach to assess the sensitivity, and the accuracy can be improved by adopting physical models using target decomposition. As this study carried out by taking crop type and stage fixed, the future work has to carry to develop a more precise relationship of even bounce, odd bounce and volume component with soil moisture for entire crop season.

\section{REFERENCES}

Gherboudj, I., Magagi, R., Aaron, A.B. and Brenda, T. (2011). Soil moisture retrieval over agricultural fields from multipolarized and multi-angular Radarsat-2 SAR data, Remote Sens. Environ., 115:33-43.

Mishra, M. D., Patel, P., Srivastava, H. S., Shukla, A., Patel, P. R. and Shukla, A.K. (2015). Approach for absolute radiometric calibration of RISAT-1 SAR data using standard target, Int. J. Remote Sens. Geosci., 4(1):28-32.

Dong, L., Baghdadi, N. and Ludwig, R. (2013). Validation of the AIEM through correlation parameterization at field scale using radar imagery in a semi-arid environment, IEEE Geosci. Remote Sens. Lett., 10(3):461-465.

Patel, P. and Srivastava H. S. (2013). Ground truth planning for synthetic aperture radar (SAR): Addressing various challenges using statistical approach, Int. J. Adv. Remote Sens. GIS Geogr., 1(2):1-17.

Patel, P. and Srivastava, H. S. (2015). An approach to validate soil moisture derived from passive microwave sensors using SAR as an interface, Int. J. Remote Sens., 36(9):2353-2374.

Patel, P., Mishra, M., Srivastava, H. S., Patel, P. R. and Sharma, Y. (2012). Comparison of linear and hybrid polarized SAR data: A case study with RISAT-1 data, National Symposium on 'Space Technology for Food \& Environmental Security', 5-7 Dec 2012, New Delhi.

Raney, R. K. (2007). Hybrid-polarity SAR architecture, IEEE Trans. Geosci. Remote Sens., 45(11):3397-3404.

Sharma, P. K., Kumar, D., Srivastava, H. S. and Patel, P. (2018). Assessment of different methods for soil moisture estimation: a review, J. Remote Sens. GIS, 9(1):57-73.
Sivasankar, T., Kumar, D., Srivastava, H. S. and Patel, P. (2018). Advances in radar remote sensing of agricultural crops: a review, Int. J. Adv. Sci. Eng. Inf. Tech., 8(4):11261137.

Srivastava, H. S. (2007). Interaction of multi-frequency multipolarized DLRESAR data with various targets: Acase study with $\mathrm{C}, \mathrm{L}$ and $\mathrm{P}$ bands acquired at all the four linear (VV, VH, HH \& HV) polarization, JEP-MWConference, pp.2:12-23, 15-16 May2007, Ahmedabad.

Srivastava, H. S., Patel, P. and Navalgund, R. R. (2006). How far SAR has fulfilled its expectation for soil moisture retrieval?,Microwave Remote Sens. Atmosph. Environ. V, Proc. of SPIE, 6410:1-12.

Srivastava, H. S., Patel, P., Manchanda, M. L. and Adiga, S. (2002). An attempt to incorporate the effect of crop cover in the soil moisture estimation using multi-incidence angle Radarsat-1 SAR data, Asian J. Geoinf., 2(1):3340.

Srivastava, H. S., Patel, P., Sharma, Y. and Navalgund R.R. (2009). Large-area soil moisture estimation using multiincidence angle Radarsat-1 SAR data, IEEE Trans. Geosci. Remote Sens., 47(8):2528-2535.

Srivastava, H. S., Sharma, P. K., Kumar, D., Sivasankar, T., Mishra, R. S., Mishra, M. andPatel, P.(2015). Soilmoisture variation over parts of Saharanpur and Haridwar districts (India) during November-2006 to June-2007 as observed bymulti-polarized(VV/HH\& VV/VH) Envisat-1 temporal SAR data, Int. J. Adv. Eng. Res. Sci., 2(1):31-39.

Srivastava, H. S., Sivasankar, T. and Patel, P. (2018a). An insight into the volume component generated from RISAT-1 hybrid polarimetric SAR data for crop biophysical parameters retrieval, ISPRS Ann. Photogramm. Remote Sens. Spatial Inf. Sci., IV-5:209-214.

Srivastava, H. S., Sivasankar, T. and Patel, P. (2018b). The sensitivity of c-band hybrid polarimetric RISAT-1 SAR data to leaf area index of paddy crop, ISPRS Ann. Photogramm. Remote Sens. Spatial Inf. Sci., IV-5:215222.

Uppala, D., Ramana, K. V., Poloju, S., Mullapudi, S. S. V. R. and Dadhwal, V. K. (2015). Rice crop discrimination using single date RISAT-1 hybrid (RH, RV) polarimetric data. Photogramm. Eng. Remote Sens., 81(7):557-563. 